\title{
Application of AI in BIM and Architecture: Base on the Example of Media Buildings
}

\author{
Yiyun Bao ${ }^{1 a^{*}}$, Yilin Cheng ${ }^{1 b}$ \\ ${ }^{a}$ College of Arts and Media, Tongji University, Shanghai, 200082, China \\ ${ }^{b}$ CATS Academy, Boston, Braintree, 02184, MA, U.S.A \\ *1850349@tongji.edu.cn
}

\begin{abstract}
Based on the background of the fourth technology revolution, architecture tends to be intelligent in the age that digital technology keeps strengthening. To research the application prospect of artificial intelligence applying in architecture, this article takes the example of media buildings, analyzes the impetus of AI to intelligence architecture, and effective improvement on design, operation, and maintenance. Besides the concept of Hypersurface, the research of features of Media Architecture is mainly based on interactivity by two-side, boundary openness, and public artistry. To research the technology support for intelligence architecture and discuss the possibility of intelligence architecture, comprehensive literature and the case of Dongdaemun Design Plaza (DDP) is analyzed, which is based on the basic theory of deep learning algorithms based on convolutional neural networks (CNN). The studies we have done suggest that the application of AI in BIM and architecture can be further extended to communities and cities, lay the foundation for the realization of Smart Cities.
\end{abstract}

Keywords: AI, BIM, intelligence architecture

\section{INTRODUCTION}

In 2009, Haeusler of Media Architecture Institute (MAI) described media architecture as facades, which make an influence in the city, with dynamic images, graphics, text[1].As a new form mixed by architecture, media, digital technology and social culture, the conception, media architecture, is still in the stage of development. American journalist and architect, Stephen Parrella, mentioned "Hypersurface" in 1998. Hypersurface is either belongs to architecture or media. It is a combination of information and space, which reflects culture adjusted by digital technology in the pioneer architecture form and set the base of architecture. In the information society, virtual information and substance have almost been combined completely. This phenomenon of combination is also strengthened by creativity.

No matter relief, mural, or painting, they all constitute the median facade of early architecture in history. The median facade plays the role of conveying information and permeating consciousness and is a part of the architecture. The two contour lines are defined by the Japanese scholar Yoshinobu Abara: the original appearance of the building as the first contour line and the temporary addition to the exterior wall as the second contour line. He believes that reducing the second contour line and merging it with the first contour line can make the city more beautiful.[2] The second contour tends to develop rapidly with the widespread existence of media architecture. Moreover, with the application of AI technology in architecture, including BIM technology, buildings are gradually showing a trend of intelligence, which conforms to the "Smart City". This article aims to research features of intelligence architecture and technology support like algorithms it use, analyzing the case of DDP. It looks forward to the further application impact on communities and urban planning of $\mathrm{AI}$ in the field of architecture, and accelerate the establishment of Smart Cities.As the earliest form which entered daily life, media architecture expresses the trend most intuitive.

\section{FEATURE OF MEDIA ARCHITECTURE}

\subsection{Interactivity by two-side}

Toyo Ito's work "Tower in the Wind" in Yokohama showed the interactive characteristics of media architecture in 1986. It adjusts the luminance and color of 
light by computer depending on the direction and speed of the wind to realize the interaction between architecture and environment. In the current consumerism environment, architecture more plays a role of medium for disseminating information expect its actual function. From static advertising image facades, to dynamic images, to the form of real-time interaction, it is becoming more and more involved in people's daily life. "If we remove all the electronic symbols on Times Square, we will find the extreme incongruity displayed on the surface of the material, because every surface of the material is helping to achieve the greatest possibility of symbol interpretation.[3]

Under the trend of intelligence architecture, the acceptance and delivery of building information are no longer one-side, and user feedback can affect the building's relative response in time. Taking art form in MORI Building Digital Art Museum as an example, formation expressed by that is not a produced by observing, but is produced in the process of human subjective interaction. Generative art and interactive art provide many designers with new inspirations and promote the presentation of avant-garde architectural concepts. Electricity, light, sound and other forms of expression stimulate multiple senses, such as vision, hearing, and touch, it enables the audience to think more about the concept of time and space. In the past, audiences seldom to have such initiative, but passively received the fixed presentation style of the buildings. Different from establishing the "Elite Image" for architects and authority for traditional media, "Elite Image" in the intelligence age has been characterized in different ways, and the media is gradually eliminating one-side authority and leading voice. With the development of intelligence architecture, the mode of two-side will become more and more perfect. The media and intelligence of buildings will also penetrate from the exterior to the interior, expanding from reality to virtual space.

\subsection{Boundary openness}

Traditional architecture is used to be closed structures, and the internal and external media buildings, the physical and virtual are no longer in a static relationship that opposes each other, but presents a compatible state, in which the boundaries become more blurred and open. With the strengthening of technology and media images in architecture, the openness is not only reflected in physical entities, but also exists between the real and virtual concepts, presenting an ambiguous state. Mobius House breaks the duo-concept of indoor and outdoor, that is, there is neither indoor nor outdoor, but a continuous deformed medium surface.[4]

\subsection{Public artistry}

Public art is the artistic creation and environmental design in a public space where the public participates freely. Its rise is based on structuralism. Its aesthetics emphasizes the intervention of the public, which produces the meaning of the work. The audience is the way to realize the value of art, and has an active role in creation, which is also an important premise for the realization of public art. It is suggested that architecture has a strong public art characteristic, and with the sudden emergence of digital art, media architecture and urban culture have further exerted this characteristic.

\section{TECHNOLOGY SUPPORT FOR INTELLIGENCE ARCHITECTURE}

After the computer has the ability to draw drawings, CG technology began to be applied to architectural design. Today, CAD is still an important auxiliary tool for architectural design. Since the 1990s, the development of Computer digital image processing and animation capabilities has also enabled it to move from tools to an expression of design ideas. In addition, the development and application of artificial intelligence have given computers more help besides design functions.

Since artificial intelligence technology is gradually applied in various industries, it has also brought a lot of inspiration to architecture. AI can help BIM achieve intelligent upgrades and promote the development of intelligence architecture. The algorithms AI used in intelligence: deep learning, neural networks, reinforcement learning, and fuzzy logic; those with a higher degree of commercialization include image recognition, natural language processing, virtual reality, etc. Justine Cassel, Chairman of the Artificial Intelligence Committee of the World Economic Forum in Davos, believes that the three keys to smart buildings are: intelligence analysis, intelligence customization, and intelligence behavior change.[5] The largest feature of AI is the ability to process massive amounts of data and make intelligence decisions. Compared with traditional computer software-assisted manpower, it has a huge advantage in effectiveness. AI can make optimal decisions based on the overall data of the project rather than a small amount of personal experience. Relying on the capabilities of artificial intelligence, such as image reignition and voice recognition, can greatly improve management efficiency and accuracy, and solve the problem of insufficient human resources. . Taking video surveillance as an example, deep learning algorithms can be used to simulate the human visual system to achieve intelligent analysis. The most widely used is the convolutional neural network, $\mathrm{CNNs}$, which can convert unstructured image data into structured object label data. After inputting data into the input layer (Figure 1), the convolution later (Figure 2) extracts and mappings 
features. The activation layer (Figure 3) activates the required feature vector. The pooling layer (Figure 4) reduces the vector size. The full connected layer (Figure 6) refits the vector to reduce the loss of feature information.

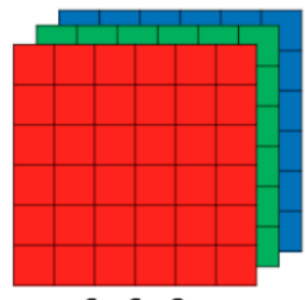

$6 \times 6 \times 3$

Figure 1 Input layer 1

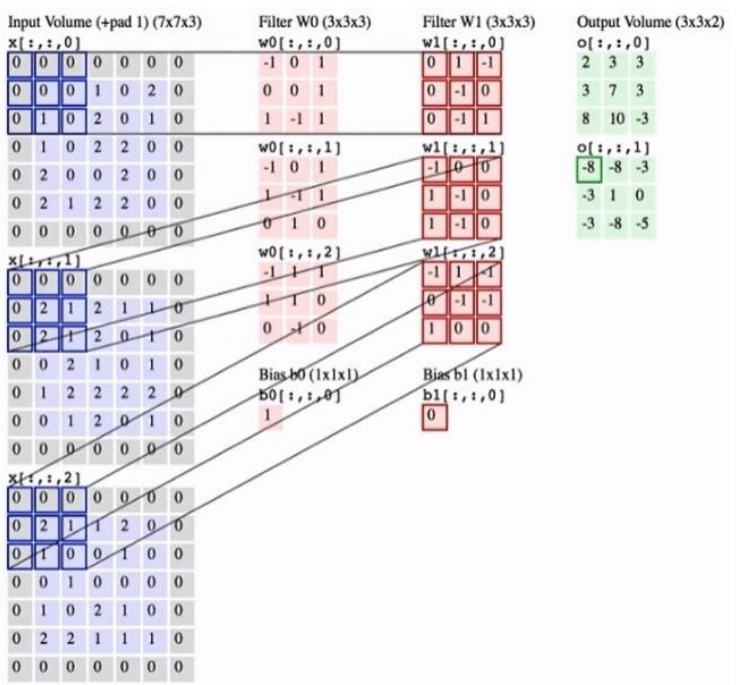

Figure 2 Convolution layer 1
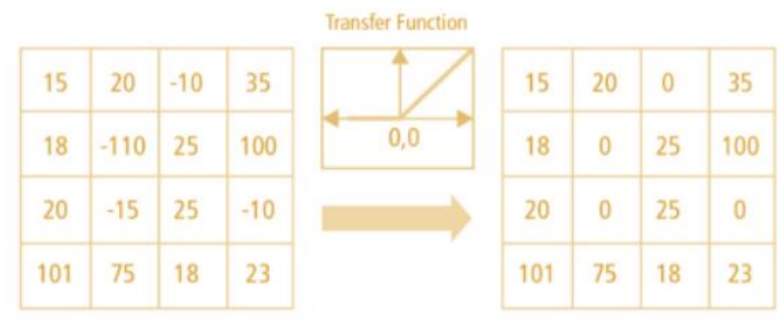

Figure 3 Activation layer 1

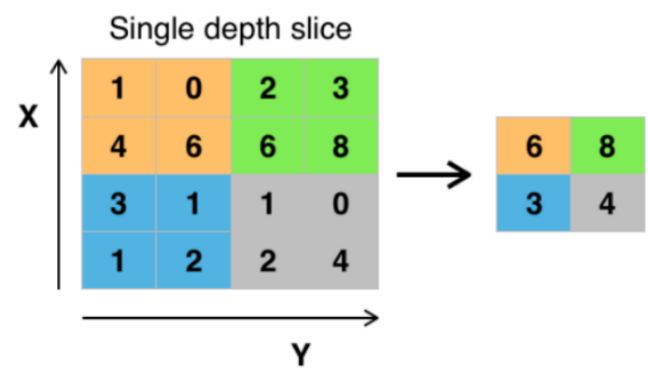

Figure 4 Pool layer 1

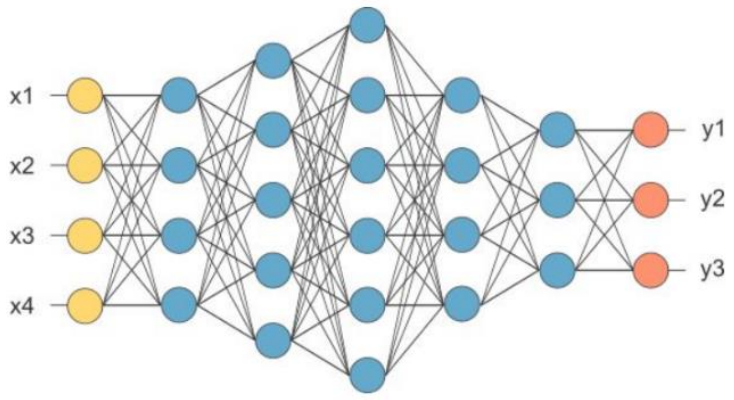

Figure 5 Full connected layer 1

Relying on big data and AI technology, the supporting role of BIM to a building can continue from the design process to the maintenance and management.[6] A building can be regarded as an ecological system. Through the analysis of it, the storage and update of the various information attributes of the building can be realized. At the same time, it can understand the understanding and needs of the public, and the efficiency and effect of building management can be improved. For the attributes of the building information, the visualization, extracting the original data for its materials, scale, and function; counting and classifying the data; then visualizing and analyzing the data, can provide more intuitive information transmission. While facilitating communication between engineers and developers, it also reduces the difficulty of understanding for users. The BIM model tool can better reflect the architecture design conception of human-oriented and assist designers in making more humane design decisions. Also, with the help of cloud collaboration functions, users can participate in this process and provide real-time feedback. Moreover, as an ecosystem, the building can be decoded for reapplication: the urban design of Las Vegas is a typical case of cultural appropriation. Venturi, a representative of postmodernism, believes that "you must learn from pop art and Las Vegas. Folk art has a collection of many valuable images." The effective management and application of BIM to building information can promote the possibility of communication and extension between technology and building.

\section{THE APPLICATION CASE OF AI IN BIM AND ARCHITECTURE}

Dongdaemun Design Plaza (DDP) in Seoul, which was decided by Zaha Hadid, interpretations combination of building and environment and application of AI in BIM and architecture. DDP is located near the oldest trading market in Seoul and combines the Korean natural landscape and traditional architecture. Combination of architecture with landscape remain the history of this city, and also safeguard the economic development at the area with a developing fashion industry. The exterior of the building uses visual illusion and multi-space mode to produce dynamic visual effects according to changes in lighting and seasonal changes, which convey different 
characteristics. The building body based on Zaha's unique natural architectural language does not match the nearby buildings, but it integrates into the city well depending on the central elements mainly based on ancient city walls and ancient cultural relics as the overall structure; moreover, the smooth curve creates the sense of architecture floating on the ground, which reduce the visually heavy feeling. The exterior landscape transforms Seoul into a green city, and the hollow and folding design on the surface shows the innovative side of architecture.

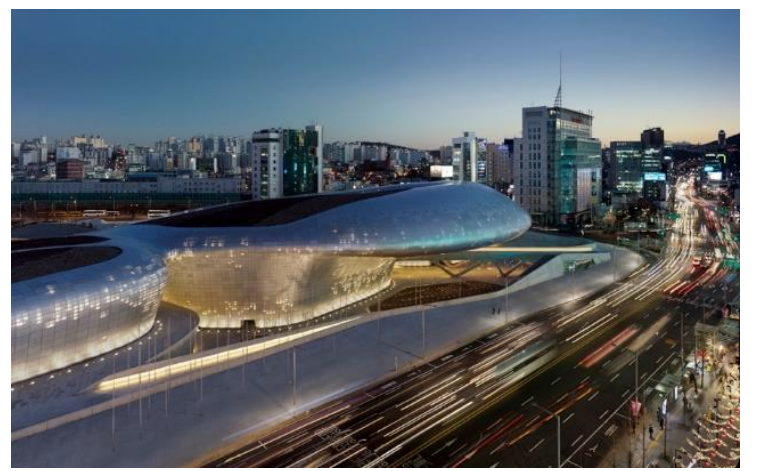

Figure 6 DDP

Benefit from the unique design of Zaha, there is not a cleaner boundary for interior and exterior of DDP. Smooth curve of exterior vague the height difference of different venue. A series of ramps, bridge-shaped structures, streamlined stairs and other elements connect spaces with different elevations and different functions. Besides the physical entities, customers' visiting sequence is also obscured. Customers do not need to follow any exact path and could also avoid repeated scenes. Consider the public requirement, DDP keeps some old elements like Illumination tower of Dongdaemun Stadium and ruins of military sites under Japanese rule. These old elements symbolize the intervention of the entire design of public. In the construction process, the advantages of BIM can be intuitively reflected. The construction of each part of the building is spatially related, not only to the design framework, but also to the spatial structure, planning requirements, and engineering technology. Taking the outer skin of DDP as an example, the stitching of about 45,000 plain surfaces with different sizes and different curvatures requires accurate calculations. [7] These large number of calculation items are difficult to achieve through traditional manual calculation methods in a short time. However, the customized BIM system combined with $\mathrm{AI}$ algorithms can calculate the model and material of each panel in a very time, and realize the control of various technical, manufacturing, and cost. BIM can be adjusted at any time during the entire design and construction process to adapt to additional site conditions, local regulations, technical requirements and cost control. In addition, the team can effectively control the design plan and various details, which is more accurate than the traditional construction process. At the same time, the owner and contractor can also have more understanding and grasp of the project.

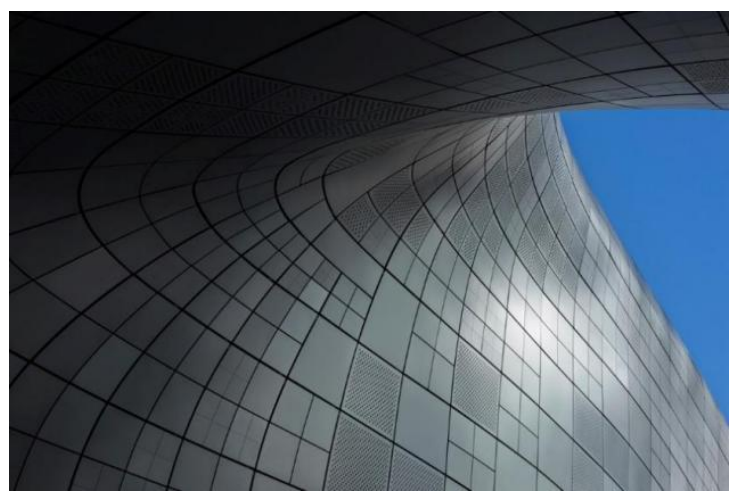

Figure 7: plain surfaces with different 1

\section{CONCLUSION}

With the support of powerful technology, architecture is showing a trend of intelligence, and the media facade is just the beginning. From the dissemination of values to the promotion of commercial advertisements, the time significance of media architecture is much more than the example above. In the paradigmatic and syntagmatic relations of different building types and functions, intelligent architectures can construct more extensions and connotations. From a long-term perspective, the application of AI in BIM and architecture can be further extended to the planning and design of communities and cities, accelerate the realization of Smart Cities, and inject vitality into the ancient construction industry. This article mainly combines theoretical foundations and specific industry cases for research, and lacks relevant experiments. If there are more abundant data statistics and specific experiments, the argumentation would be more comprehensive.

\section{ACKNOWLEDGMENT}

Firstly, I would like to show my deepest gratitude to Prof. Lio, who provided me with valuable guidance in every stage of the writing of this thesis. Further, I would like to thank all my friends and parents for their encouragement and support. Without all their enlightening instruction and impressive kindness, I could not have completed this thesis. I would like to expressing gratitude to all the teachers who help me complete the essay. Also, I want to thank all my friends and family who provide me support during the period of time.

\section{REFERENCES}

[1]Ashihara Yoshinobu. The Aesthetic Townscape. Baihua Literature and Art Publishing House. 2006

[2]Cristina, Giusppa(ed.): 2001, Architecture and Science. Wiley-Academy, London, P190 
[3]Gaofeng Mi, Peng Zhao. Study on IP Cross-media Publishing Strategy of Tencent Interactive Entertainment[J]. A Vast View on Publishing, 2017(15): 57-59

[4]Zellner. P. 1999, Hybrid Space, Thames \& Hudson, London, P46

[5]Justine Cassel, 2017, Alibaba intelligent building (IB) summit

[6]LENG Shuo, HU Zhenzhong, (2018) A Review of BIM-Based Artificial Intelligence Methods, Journal of Graphics, 39(5)

[7]Kim, Seung-Bum. "The impact of'Zaha Hadid'and her design on the public discourse about Dongdaemun Design Plaza." Journal of the Architectural Institute of Korea Planning \& Design 30.10 (2014): 35-44. 